Notfall Rettungsmed 2012 · 15:657-657

DOI 10.1007/s10049-011-1538-5

Online publiziert: 9. November 2012

(c) Springer-Verlag Berlin Heidelberg 2012

\author{
R. Stern ${ }^{1} \cdot$ H.-R. Arntz ${ }^{2}$ \\ ${ }^{1}$ Abt. Innere Medizin, Zentrum für Herzinsuffizienz, Jüdisches Krankenhaus Berlin \\ ${ }^{2}$ Medizinische Klinik II, Kardiologie und Pulmonologie, Charite - Universitätsmedizin Berlin, \\ Campus Benjamin Franklin, Berlin
}

\title{
Ethik und Notfallmedizin
}

\section{》 „Was ist ein guter Tod?"}

dizin sind ethische Fragen so allgegenwärtig wie in der Notfallmedizin. Die Eckpunkte dieses weiten Felds sind begrenzte Ressourcen - man denke an die Situation des Massenanfalls von Patienten bei einem schweren Unfallereignis. Dass der Begriff der „Triage“ in diesem Zusammenhang verlassen wurde, löst im konkreten Fall keins der Probleme. Ein anderer Eckpunkt ist die Notwendigkeit, Sofortentscheidungen treffen zu müssen - klassisch bei der Entscheidung, einen Reanimationsversuch zu beginnen oder zu unterlassen.

\section{D) Schwierig sind \\ Entscheidungen am Lebensende bei schwer kranken Patienten}

Problematisch sind auch Entscheidungen am Lebensende bei schwer kranken Patienten und adäquates Vorgehen bei chronisch bettlägerigen, oft multipel erkrankten oder dementen Patienten in Heimen. Die notwendigen Entscheidungen werden oft dadurch erschwert, dass verlässliche Hintergrundinformationen über den Patienten fehlen. Aber auch andere Aspekte können und werden bewusst oder unbewusst auf die Entscheidungen Einfluss nehmen, wie z. B. der weltanschauliche oder religiöse Hintergrund der Rettungskräfte. In diesem Heft haben wir uns einiger dieser Fragen angenommen, nicht um sie pauschal und endgültig zu beantworten - dieser Versuch müsste schon an der Einzigartigkeit jeder einzelnen Entscheidungssituation scheitern - sondern um ein Nachdenken über einige typische Probleme anzuregen und vielleicht sogar einige Wege zu Lösungsansetzen aufzuzeigen.
Prof. A. Steinberg, Rabbiner und Kinderneurologe aus Jerusalem, macht sich Gedanken über die Frage „Was ist ein guter Tod?", etwas was wir jedem unserer Patienten und natürlich auch uns selbst wünschen. Selbstverständlich kann es auf diese Frage keine pauschale Antwort geben, wissen wir doch von unseren Patienten, wie unterschiedlich die Vorstellungen hierzu sind, auch wenn die Gemeinsamkeit der Angst vor Schmerzen besteht. Gibt es Bedingungen, unter denen der Rettungsdienst selbst bei erkennbar zu Ende gehendem Leben nicht mehr gerufen werden sollte? Dieses Thema berührt die Frage der rechtzeitigen Auseinandersetzung mit dem näher rückenden eigenen Tod, ein Thema das gerne vom Patienten selbst, den Angehörigen, aber auch den die Patienten langfristig betreuenden Ärzten vermieden wird. An Kliniken eingerichtete Ethik-Komitees können bei entsprechender Vorsorge selbst für akute „Adhoc"-Probleme verfügbar sein und den verantwortlichen Ärzten Hilfe und Unterstützung bei ihren unter Zeitdruck zu fällenden schwierigen Entscheidungen sein. Prinzipiell ist vorstellbar, dass solche Komitees auch für den Rettungsdienst eingerichtet werden, um allerdings dann meist im Nachhinein - „post hoc“ - den Rettungskräften mit Rat und Tat zur Seite zu stehen. Prof. K. Wegscheider aus Hamburg, setzt sich mit der Fragestellung „Was ist Aussichtslosigkeit" in der prognostischen Beurteilung auseinander. Zunächst glaubt man das, was im englischen als ,Futility" bezeichnet wird, relativ gut beurteilen zu können. Tatsächlich handelt es sich aber, wie Wegscheider nachweist, objektiv um ein fast unlösbares Problem, dass letzt- endlich auf eine ethische Frage hinausläuft, die der verantwortliche Arzt beantworten muss, wie der Autor in einem persönlichen abschließenden Zusatz zu seiner Analyse unterstreicht.

Wir wünschen uns eine interessierte Leserschaft und hoffen, Ihnen wertvolle Anregungen für Ihren medizinischen Alltag gegeben zu haben.

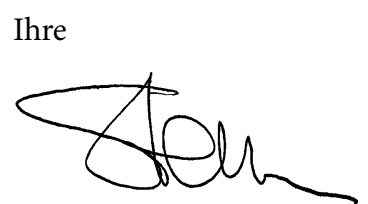

Richard Stern

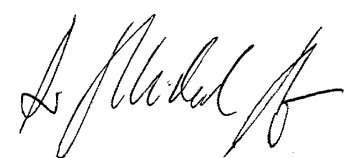

Hans-Richard Arntz

\section{Korrespondenzadresse}

\section{Dr. R. Stern}

Abt. Innere Medizin, Zentrum für Herzinsuffizienz, Jüdisches Krankenhaus Berlin Heinz-Galinski-Straße 1, 13347 Berlin

Richard.Stern@jkb-online.de

\section{Prof. Dr. H.-R. Arntz}

Medizinische Klinik II, Kardiologie und Pulmonologie, Charite - Universitätsmedizin Berlin, Campus Benjamin Franklin Hindenburgdamm 30, 12200 Berlin hans-richard.arntz@charite.de 